\title{
A NON-ABELIAN VARIATION ON THE SAVVIDY VACUUM OF THE YANG-MILLS GAUGE THEORY*
}

\author{
Suzhou Huang \\ Department of Physics, FM-15, University of Washington \\ Seattle, Washington 98195 \\ and Center for Theoretical Physics, Laboratory for Nuclear Science, and Department of Physics \\ Massachusetts Institute of Technology, Cambridge, Massachusetts 02139
}

\author{
A. R. Levi \\ Department of Physics, Boston University \\ 590 Commonwealth Avenue, Boston, MA 02215
}

\begin{abstract}
BU-HEP-93-22
CTP 2267

(December 1993)

As a prelude to a truly non-perturbative evaluation of the effective potential in terms of lattice $\mathrm{QCD}$, the one loop effective potential for a non-Abelian gauge configuration is calculated using the background field method. Through a non-trivial correlation between the space and color orientations the new background field avoids the possible coordinate singularity, Det $B_{i}^{a}=0$, observed recently by Ken Johnson and his collaborators in their Schrödinger functional study of the SU(2) Yang-Mills theory. In addition, since our ansatz generates a constant color magnetic field through the commutator terms rather than derivative terms, many of the technical drawbacks the Savvidy ansatz suffers on a lattice can be avoided. Our one loop study yields qualitatively the same result as that of Savvidy's.
\end{abstract}

${ }^{*}$ This work is supported in part by funds provided by the U. S. Department of Energy (D.O.E.) under contracts \#DE-FG0688ER40427, \#DE-AC02-76ER03069 and \#DE-FG02-91ER40676 


\section{INTRODUCTION}

Even after many years of intensive efforts, the vacuum structure of non-Abelian gauge theories still remains elusive to us. Due to the lack of other systematic methods, the conventional approach is to analyze the effective action using the so-called background field method. In their pioneer work [1] Savvidy and Matinyan, analogous to the case of QED, calculated up to one loop order the effective potential for an Abelian background gauge field,

$$
A_{\mu}^{a}(x)=\frac{1}{2} H \delta^{a 3}\left(x_{1} \delta_{\mu 2}-x_{2} \delta_{\mu 1}\right),
$$

which generates a constant color magnetic field $B_{i}^{a}=H \delta_{i 3} \delta_{a 3}$ in the $\mathrm{SU}(2)$ Yang-Mills theory. It was then found, remarkably, that the vacuum with this background field is energetically favored over the perturbative vacuum $(H=0)$. Unfortunately, a more careful analysis [2] soon revealed that there exists an imaginary part in the effective potential, indicating that the background field Eq.(1.1) is not a minimum but rather a saddle point in the configuration space in the context of the loop expansion. The subsequent work by the Copenhagen group [3], still within the framework of loop expansion, tried to remedy the physically appealing picture of Savvidy's vacuum by introducing inhomogeneouty, leading to the so-called Copenhagen vacuum.

However, the validity of the loop expansion has been questioned by Maiani et al [4]. These authors strongly argued that the calculation of the effective potential in the presence of a background field is actually non-perturbative. So far the only known non-perturbative technique in field theory that can be made systematic is the lattice approach. More recently several works with the lattice approach have appeared in the literature, both in four dimensions [5] and three dimensions [6]. Due to various technical reasons, mainly related to the fact that the Savvidy ansatz in Eq.(1.1) is non-uniform, all these lattice works have yet to yield a conclusive result.

Recently, in an unrelated but very interesting work by Johnson and his collaborators [7], the Schrödinger functional approach in terms of magnetic field strength is applied to the $\mathrm{SU}(2)$ Yang-Mills gauge theory. In the explicit Schrödinger functional Hamiltonian they find that there is a factor $1 / \operatorname{Det} B_{i}^{a}$ in the kinetic energy term, similar to the $1 / r$ factor in the quantum mechanics Schrödinger equation in polar coordinate. This factor may signal a potential coordinate singularity for the color magnetic field in the configuration space, similar to that of the wavefunctions in quantum mechanics have to satisfy certain boundary conditions at $r=0$. Since the Savvidy ansatz yields Det $B_{i}^{a}=0$, due to its intrinsic Abelian nature, it is desirable to seek an alternative background field, which avoids this potential coordinate singularity. A simple choice for $\mathrm{SU}(2)$ gauge group, suggested to us by Ken Johnson, is the following

$$
A_{0}^{a}(x)=0, \quad \text { and } \quad A_{i}^{a}(x)=h \delta_{i}^{a},
$$

where $h$ is a constant in space-time. The corresponding color magnetic field is simply given by $B_{i}^{a}=g h^{2} \delta_{i}^{a}$ and obviously has $\operatorname{Det} B_{i}^{a} \neq 0$ when $h \neq 0$.

It is easy to recognize that the constant magnetic field is generated by Eq.(1.2) through the commutator terms in the field strength, rather than the derivative terms as in the Savvidy's case. It has been proved [8] that there are no other ways to generate a constant magnetic field. Due to the non-trivial correlation between its directions in space and color space, the background field Eq.(1.2) evades the possible coordinate singularity. In addition, since the non-Abelian background is space-time independent, it is much easier to put it on a lattice than the non-constant background field Eq.(1.1). For example, the periodic boundary condition is automatic.

Before we launch an extensive numerical simulation, it is still worthwhile to calculate the one-loop effective potential for the non-Abelian background field Eq.(1.2). Even though it is not totally trustworthy, the loop expansion can still provide indicative information. Furthermore, to examine whether the qualitative feature of the one-loop effective potential strongly depends on the choice of the background field is also interesting. In particular, we would like to find out whether the coordinate singularity has any connection with the existence of the imaginary part in the effective potential. In this paper we will only address the question of the one-loop calculation and leave the lattice study for the future.

In doing the one-loop calculation we encountered several technical points, which were known in the literature but not emphasized enough. We decide to briefly go through (in the next section) the standard background field method in gauge theories, with those technical points in mind. In section III we explicitly calculate the one-loop effective potential for the non-Abelian gauge background in the three dimensional SU(2) Yang-Mills gauge theory. Unfortunately, we were unable to carry out a similar calculation in four dimensions (except the divergent part, which is done in the appendix), due to technical difficulties in evaluating certain integrals. Finally, we summarize and briefly discuss the generalization of the lattice background field method in section IV. 


\section{FORMALISM}

In this section we outline the basic steps in the background field method in gauge theories 9]. There are two reasons to go through the well-known method. The first is to establish our own notations. The second is to emphasize two technical aspects, the question of whether it is necessary to require the background field satisfy the classical equation of motion and the gauge choice in the evaluation of the functional integral. When possible we follow the convention of Abbott [9].

The generating functional in the background field method (in Euclidean space) starts with

$$
\tilde{Z}[J, A]=\int[d Q] \operatorname{det}\left[\frac{\delta G^{a}}{\delta \omega^{b}}\right] \exp \left\{-\int d^{d} x\left[\mathcal{L}(A+Q)+J_{\mu}^{a} Q_{\mu}^{a}\right]\right\} \prod_{x, a} \delta\left[G^{a}\right]
$$

where $G^{a} \equiv \partial_{\mu} Q_{\mu}^{a}+g f^{a b c} A_{\mu}^{b} Q_{\mu}^{c}=D_{\mu}^{a b}(A) Q_{\mu}^{b}$ is the background field gauge condition and det $\left[\delta G^{a} / \delta \omega^{b}\right]$ is the corresponding Jacobian. It is clear from the above definition the background field $A_{\mu}^{a}$ is fixed and should be regarded as an external parameter in the process of doing the $Q$-integral. Note also that we do not exponentiate the gauge constraint $\prod_{x, a} \delta\left[G^{a}\right]$, in contrast with what was usually done. The necessity of enforcing the gauge constraint with explicit delta-functions in Eq.(2.1) will be discussed in detail soon.

It is easy to verify that $\tilde{Z}[J, A]$ is invariant under the infinitesimal gauge transformations

$$
\begin{gathered}
\delta A_{\mu}^{a}=-f^{a b c} \omega^{b} A_{\mu}^{c}+\frac{1}{g} \partial_{\mu} \omega^{a} ; \\
\delta J_{\mu}^{a}=-f^{a b c} \omega^{b} J_{\mu}^{c} .
\end{gathered}
$$

As a consequence of this invariance, the effective action in the background field method, defined as the Legendre transform of $\tilde{Z}$,

$$
\tilde{\Gamma}[\tilde{Q}, A]=-\ln \tilde{Z}[J, A]+\int d^{d} x J_{\mu}^{a} \tilde{Q}_{\mu}^{a}
$$

with $\tilde{Q}_{\mu}^{a}=\delta \ln \tilde{Z} / \delta J_{\mu}^{a}$, is invariant under

$$
\begin{gathered}
\delta A_{\mu}^{a}=-f^{a b c} \omega^{b} A_{\mu}^{c}+\frac{1}{g} \partial_{\mu} \omega^{a} \\
\delta \tilde{Q}_{\mu}^{a}=-f^{a b c} \omega^{b} \tilde{Q}_{\mu}^{c} .
\end{gathered}
$$

As shown by Abbott [9], when $\tilde{Q}_{\mu}^{a}=0, \tilde{\Gamma}[0, A]$ coincides with the usual effective action. Since Eq. 2.4) is a pure gauge transformation when $\tilde{Q}$ vanishes, $\tilde{\Gamma}[0, A]$ must be a gauge invariant functional of $A_{\mu}^{a}$.

A standard way to evaluate $\tilde{Z}[J, A]$ explicitly is to make a loop expansion. For the purpose of convenience, we exponentiate the Jacobian $\operatorname{det}\left[\delta G^{a} / \delta \omega^{b}\right]$ and gauge constraints $\prod_{x, a} \delta\left[G^{a}\right]$ by introducing the Faddeev-Popov ghost fields $\left(\theta^{a}\right.$ and $\left.\bar{\theta}^{a}\right)$ and a real scalar auxiliary field $\sigma^{a}$ respectively,

$$
\begin{aligned}
\tilde{Z}[J, A]= & \int[d Q][d \theta][d \bar{\theta}][d \sigma] \exp \left\{-\int d^{d} x\left[\mathcal{L}(A+Q)+\bar{\theta}^{a} D_{\mu}^{a c}(A) D_{\mu}^{c b}(A) \theta^{b}+\right.\right. \\
& \left.\left.+2 i \sigma^{a} D_{\mu}^{a b}(A) Q_{\mu}^{b}+J_{\mu}^{a} Q_{\mu}^{a}\right]\right\} .
\end{aligned}
$$

If we only want to calculate $\tilde{Z}[J, A]$ to one-loop order, then it is equivalent to evaluate Eq.(2.5) in the steepest descent approximation,

$$
\begin{aligned}
\tilde{Z}[J, A] \approx & \tilde{Z}_{1}[J, A] \equiv \int[d Q][d \theta][d \bar{\theta}][d \sigma] \exp \left\{-\int d^{d} x\left[\mathcal{L}(A)+\mathcal{L}^{(1)}(A, Q)+\right.\right. \\
& \left.\left.\mathcal{L}^{(2)}(A, Q)+\bar{\theta}^{a} D_{\mu}^{a c}(A) D_{\mu}^{c b}(A) \theta^{b}+2 i \sigma^{a} D_{\mu}^{a b}(A) Q_{\mu}^{b}+J_{\mu}^{a} Q_{\mu}^{a}\right]\right\}
\end{aligned}
$$


where

$$
\begin{gathered}
\mathcal{L}^{(1)}(A, Q)=-Q_{\mu}^{a} D_{\mu}^{a b}(A) F_{\mu \nu}^{b}(A) ; \\
\mathcal{L}^{(2)}(A, Q)=Q_{\mu}^{a}\left[-\frac{1}{2}\left(D_{\rho}(A) D_{\rho}(A)\right)^{a b} \delta_{\mu \nu}+i g F_{\mu \nu}^{a b}(A)\right] Q_{\nu}^{b} \equiv Q_{\mu}^{a} M_{\mu \nu}^{a b}(A) Q_{\nu}^{b} .
\end{gathered}
$$

In arriving at the expression of $\mathcal{L}^{(2)}(A, Q)$, the gauge constraint condition $D_{\mu}^{a b} Q_{\mu}^{b}=0$ has been used.

We can now do the $Q$-integral in $\tilde{Z}_{1}[A, J]$ and make the Legendre transform as in Eq.(2.3). By setting $\tilde{Q}_{\mu}^{a}=0$ the final expression for the effective action to one-loop order can be written as $\Gamma[A]=-\ln \tilde{Z}_{1}[A]$, with

$$
\begin{aligned}
\tilde{Z}_{1}[A]= & e^{-\int d^{d} x \mathcal{L}(A)} \cdot \operatorname{det}[-D(A) D(A)] \cdot \\
& \int[d Q][d \sigma] \exp \left\{-\int d^{d} x\left[Q_{\mu}^{a} M_{\mu \nu}^{a b}(A) Q_{\nu}^{b}+2 i \sigma^{a} D_{\mu}^{a b}(A) Q_{\mu}^{b}\right]\right\} \\
= & e^{-\int d^{d} x \mathcal{L}(A)} \cdot \operatorname{det}[-D(A) D(A)] \cdot\left\{\operatorname{det} M_{\mu \nu}^{a b} \cdot \operatorname{det} N^{a b}\right\}^{-1 / 2},
\end{aligned}
$$

with $N^{a b}=-D_{\mu}^{a c}\left(M^{-1}\right)_{\mu \nu}^{c e} D_{\nu}^{e b}$. The first factor is the classical contribution. The second and third factors are the one-loop quantum corrections due to the ghost field $(\theta)$ and fluctuation field $(Q)$, respectively. Notice that the linear term $\mathcal{L}^{(1)}(A, Q)$ drops out automatically in the calculation process. There is no need to require the background field to satisfy the classical equation of motion $D_{\mu}^{a b}(A) F_{\mu \nu}^{b}(A)=0$. This independence of the linear term remains true to all orders in loop expansion, because the effective potential is a sum of all one-particle irreducible diagrams with $A$ fields on the external lines and $Q$ fields on internal lines (when $\tilde{Q}_{\mu}^{a}=0$ ). In fact, the linear term is always compensated by the source $J_{\mu}^{a}$. Remember that the physical limit in the background field method is $\tilde{Q}_{\mu}^{a}=0$, not $J_{\mu}^{a}=0$.

At this point we would like to comment on the implementation of the gauge condition $\prod_{x, a} \delta\left[G^{a}\right]$ in Eq. (2.1). The standard approach is to exponentiate this factor,

$$
\tilde{Z}[J, A ; \alpha]=\int[d Q] \operatorname{det}\left[\frac{\delta G^{a}}{\delta \omega^{b}}\right] \exp \left\{-\int d^{d} x\left[\mathcal{L}(A+Q)-\frac{1}{2 \alpha}\left(G^{a}\right)^{2}+J_{\mu}^{a} Q_{\mu}^{a}\right]\right\},
$$

where $\alpha$ is the gauge fixing parameter. In order to write Eq.(2.1) into the form of Eq.(2.10), one has to generalize the gauge condition as $\prod_{x, a} \delta\left[G^{a}-f^{a}\right]$, with $f^{a}$ being arbitrary, and then to integrate out $f^{a}$ with the weighting factor $\exp \left[-\left(f^{a}\right)^{2} / 2 \alpha\right]$. If $\tilde{Z}[J, A]$ with the generalized gauge condition were independent of $f^{a}$, as in the case of zero background field $A_{\mu}^{a}=0, \tilde{Z}[J, A ; \alpha]$ would be independent of the gauge parameter $\alpha$ and therefore the exact equivalence between Eq.(2.1) and Eq.(2.10). However, in the presence of non-vanishing background field $A_{\mu}^{a}, \tilde{Z}[J, A]$ in general would depend on $f^{a}$, as can be easily seen in Eq.(2.5) by adding a term $2 i \sigma^{a} f^{a}$ to the exponential. Physically, this dependence on $f^{a}$ is natural, because there would exist correlations between $f^{a}$ and certain combinations of the background field, such as $\left(D_{\mu}^{a b}(A) f^{b}\right)^{2}$ when $A_{\mu}^{a}$ is non-vanishing. If indeed $\tilde{Z}[J, A]$ depended on $f^{a}$, then the integral

$$
\left.\tilde{Z}[J, A ; \alpha] \equiv \int[d f] \tilde{Z}[J, A]\right|_{G^{a}=f^{a}} \cdot \exp \left\{-\frac{1}{2 \alpha}\left(f^{a}\right)^{2}\right\}
$$

would have to depend on $\alpha$ in general, which in turn implies that Eq.(2.10) could not be equivalent to Eq.(2.1) at all times.

This gauge dependence of the effective action calculation has long been recognized in the literature [10]. It was argued by Vilkovisky that the correct choice of the gauge parameter is to take $\alpha \rightarrow 0$ limit, or to choose the Landau background field gauge. Since $\exp \left[-x^{2} / 2 \alpha\right] \propto \delta(x)$ in the limit of $\alpha=0$, Eq.(2.10) is formally equivalent to Eq.(2.1) in the Landau background field gauge. It should be emphasized that Eq.(2.10) would still define a gauge invariant effective action with respect to the background field $A_{\mu}^{a}$. It is only the functional form of the effective action that depends on the gauge parameter when $\alpha \neq 0$. Finally, in the context of finite temperature QCD, this gauge dependence has been emphasized by Hansson and Zahed [11]. 


\section{EFFECTIVE POTENTIAL}

In this section we apply the background field method to calculate the effective potential for the background field mentioned in the Introduction in three dimensional SU(2) Yang-Mills gauge theory in Euclidean space. The three dimensional analog of Eq.(1.2) would be,

$$
A_{\mu}^{a}(x)=h \delta_{a \mu}
$$

This choice of $A_{\mu}^{a}$ leads to non-vanishing field strengths, through commutator terms rather than derivative terms as in the case of Savvidy's ansatz,

$$
B_{\mu}^{a} \equiv \frac{1}{2} \epsilon_{\mu \alpha \beta} F_{\alpha \beta}^{a}(x)=g h^{2} \delta_{a \mu},
$$

with $g$ being the coupling constant. Due to the non-trivial correlation between the color and space-time orientations the determinant $\operatorname{det} B_{\mu}^{a} \neq 0$ when $h \neq 0$. Notice that in Euclidean space all components of the field strength are magnetic-like.

Since the background field is a constant in space-time it is convenient to work in momentum-space. Using the formula $\operatorname{det} M=\exp (\operatorname{Tr} \ln M)$ combined with Eq.(2.9), we have the expression for the effective potential to one-loop order in our ansatz

$$
V_{\text {eff }}(h)=\mathcal{L}(h)-\int \frac{d^{3} p}{(2 \pi)^{3}} \ln G(h ; p)+\frac{1}{2} \int \frac{d^{3} p}{(2 \pi)^{3}}\{\ln M(h ; p)+\ln N(h ; p)\},
$$

where

$$
\begin{gathered}
G(h ; p) \equiv \operatorname{det}\left[-D_{\mu}^{a c}(h ; p) D_{\mu}^{c b}(h ; p)\right]=\left(p^{2}+2 m^{2}\right)\left(p^{4}+4 m^{4}\right) \\
M(h ; p) \equiv \operatorname{det}\left[M_{\mu \nu}^{a b}(h ; p)\right]=p^{6}\left(p^{2}-4 m^{2}\right)\left(p^{4}+6 m^{2} p^{2}+16 m^{4}\right)\left(p^{6}+4 m^{2} p^{4}-16 m^{6}\right) \\
N(h ; p) \equiv \operatorname{det}\left[-D_{\mu}^{a c}(h ; p)\left(M^{-1}\right)_{\mu \nu}^{c e}(h ; p) D_{\nu}^{e b}(h ; p)\right] \\
=\frac{\left(p^{6}-4 m^{6}\right)\left(p^{4}+2 m^{2} p^{2}+4 m^{4}\right)}{\left(p^{4}+6 m^{2} p^{2}+16 m^{4}\right)\left(p^{6}+4 m^{2} p^{4}-16 m^{6}\right)}
\end{gathered}
$$

with $m \equiv g h$. Non-positive definiteness of $M(h ; p)$ and $N(h ; p)$ for small $p^{2}$ indicates that the background field in Eq.(3.1) is only a saddle point in configuration space and therefore is not stable. To reveal the physical origin of the existence of the unstable modes, let us explicitly write

$$
M_{\mu \nu}^{a b}(h ; p)=\frac{1}{2}\left[\left(p^{2}+2 g^{2} h^{2}\right) \delta^{a b} \delta_{\mu \nu}+2 i g h \epsilon^{a b c} p_{c} \delta_{\mu \nu}+2 g^{2} h^{2} \epsilon^{a b c} \epsilon_{c \mu \nu}\right]
$$

which is a $9 \times 9$ matrix for a given momentum $p$. This matrix can be interpreted as the Hamiltonian of a relativistic spin-1 $\left(\vec{\sigma}_{s}\right)$ and color spin-1 $\left(\vec{\sigma}_{c}\right)$ boson, with the first term being the free particle part, the second the $\vec{p} \cdot \vec{\sigma}_{c}$ and the third $\vec{\sigma}_{s} \cdot \vec{\sigma}_{c}$. The last two terms can be negative for low momentum, depending on the relative orientations of $\vec{p}, \vec{\sigma}_{s}$ and $\vec{\sigma}_{c}$. It is interesting to observe that in the case of Savvidy's ansatz the corresponding $M$ matrix is the Landau diamagnetic Hamiltonian.

Using the regularization of Salam and Strathdee [12], which is a variation of Schwinger's proper time method [13, the integrals in Eq.(3.3) can be worked out explicitly. Because the three dimensional Yang-Mills theory is super renormalizable, the only divergence we encounter is an overall additive constant. The regularization procedure includes three steps. Firstly, an integral representation for logarithmic function is used. For real $E$, positive or negative,

$$
\ln (E-i \delta)=\frac{1}{\epsilon}-\frac{i^{\epsilon}}{\epsilon \Gamma(\epsilon)} \int d t t^{\epsilon-1} e^{-i t(E-i \delta)},
$$

in the limit of $\epsilon \rightarrow 0^{+}$. The $i \delta$ in Eq.3.8) with $\delta \rightarrow 0^{+}$is to ensure the convergence of the integral. When $E$ is explicitly complex, Eq.(3.8) can be easily generalized. Then the momentum integration can be done using

$$
\int \frac{d^{3} p}{(2 \pi)^{3}} e^{-i t p^{2}}=\frac{1}{8 \pi^{3 / 2}}(i t)^{-3 / 2}
$$


The remaining $t$-integral can be converted into a Gamma function through the contour integral technique. Finally, the limit of $\epsilon \rightarrow 0$ is taken.

After dropping an overall divergent constant, the effective potential has the following expression,

$$
V_{\mathrm{eff}}(h)=\frac{3}{2} g^{2} h^{4}-\frac{2-\sqrt{2}}{3 \pi} g^{3} h^{3}-i \frac{5}{6 \pi} g^{3} h^{3},
$$

or if we define $B \equiv g h^{2}$,

$$
V_{\mathrm{eff}}(B)=\frac{3}{2} B^{2}-\frac{2-\sqrt{2}}{3 \pi}(g B)^{3 / 2}-i \frac{5}{6 \pi}(g B)^{3 / 2} .
$$

Again the first term in Eq. 3.10 ) is the classical contribution, the second term is the ghost field $(\theta)$ contribution and the third is the fluctuation field $(Q)$ contribution. Compared to the calculation of Trottier [14] in the Savvidy ansatz, we find qualitative similarity between the two results. If we ignore the imaginary part in the effective potential temporarily, we would find a non-trivial minimum,

$$
h_{\min }=\frac{2-\sqrt{2}}{6 \pi} g,
$$

or

$$
B_{\min }=\left(\frac{2-\sqrt{2}}{6 \pi}\right)^{2} g^{3} .
$$

Since the effective potential evaluated at the minimum point is the energy density relative to the perturbative vacuum, $V_{\text {eff }}\left(h_{\min }\right)<0$ signals that the system prefers to spontaneously generate color magnetic fields in order to gain energy and in turn signals the instability of the perturbative vacuum. However, due to the presence of the imaginary part in $V_{\text {eff }}$, the vacuum characterized by Eq.(3.11) itself can not be stable either, at least not in the loop expansion to one-loop order.

In passing, if the Feynman gauge $(\alpha=1)$ were used, which would be equivalent to ignoring the term $\ln N(h ; p)$ in Eq.(3.3), we would have obtained

$$
\left.V_{\text {eff }}(h)\right|_{\alpha=1}=\frac{3}{2} g^{2} h^{4}-C_{R} g^{3} h^{3}-i C_{I} g^{3} h^{3},
$$

with $C_{R}=0.43514 \ldots$ and $C_{I}=0.26989 \ldots$ It is possible to get exact expressions for $C_{R}$ and $C_{I}$. But these expressions are complicated and not illuminating. We will not bother to write them down here. Nevertheless, it is sufficient to mention that the values of $C_{R}$ and $C_{I}$ are different from their corresponding counterparts in Eq.(3.10).

To generalize the above calculation to four dimensions, we could try the ansatz $A_{i}^{a}=h \delta_{i a}(i=1,2,3)$ in the temporal gauge $A_{0}^{a}=0$, as in Eq.(1.2). In fact, such a calculation already exists in the literature [15] about one decade ago, with a different motivation. However, the minimum point found in that work is not renormalization group invariant, which in turn implies that their $\beta$-function differs from the correct $\mathrm{SU}(2)$ value (by a factor of 2 ). This is in contradiction with rather general arguments, for example in 沟, that the ultraviolet behaviors of the Yang-Mills theory stays the same, independent of the presence or absence of a background field, since the background field is only relevant in the infrared regime. Furthermore, in the work of Lüscher, in terms of Hamiltonian approach [16], and of Kolker and van Baal, in terms of Lagrangian approach [17], where they calculated the coefficients of the effective potential in $\mathrm{SU}(2)$ Yang-Mills theory for constant background field up to fourth order in the background field on a finite torus, the $\beta$-function was found not to be modified by the presence of the background field. The same conclusion was shown [18] to hold explicitly at one loop level, at least for some classes of background fields, without expanding the effective potential as a power series of the background fields. Since the $\beta$-function is gauge ( or $\alpha$ ) independent in the dimensional regularization and minimal subtraction scheme [19], although the finite part is explicitly $\alpha$ dependent, we suspect that the authors of reference [15] overlooked certain subtleties in calculating the four dimensional functional determinant. Due to the lack of details in their paper we could not explicitly check their results. However, since the imaginary part of the effective potential is finite and easy to calculate, we have verified that the authors of [15] missed a term due to one of their $\lambda_{4,5,6,7}$ in Eq.(5), which could become negative for low enough momenta. To find out what really happens in our case, we will show, in the appendix, that the $\beta$-function is not affected in the presence of the background field Eq.(1.2) through an explicitly calculation of the divergent part of the effective potential. 


\section{SUMMARY AND DISCUSSION}

To summarize, we have calculated the effective potential for a particularly chosen non-Abelian background field in the three dimensional SU(2) Yang-Mills theory. Technical questions related to the linear term and the gauge choice were illustrated. The result is found to be qualitatively similar to that of the Abelian ansatz of the Savvidy type [14], both in real part, which indicates a spontaneous generation of the color magnetic field, and imaginary part, which signals the instability of the background field Eq.(3.1) as the vacuum configuration under the loop expansion. Though not explicitly calculated, the four dimensional effective potential with the same non-Abelian ansatz in the temporal gauge is expected to behave very much the same. Given the qualitative similarity between the three dimensional and four dimensional effective potential in the Savvidy ansatz, we suspect that the effective potential is insensitive to the coordinate singularity, Det $B_{i}^{a}=0$, if it indeed exists.

It is important to recognize that the starting point in Eq.(2.1) would be a well defined problem if the functional were evaluated non-perturbatively. The appearance of the imaginary part in the effective potential is only caused by the loop expansion. In other words, when we were doing steepest descent approximation, we were expanding at a saddle point. In addition, even if the expansion point were a true minimum, the stationary solution of the effective potential calculated up to a finite loop order could not be trusted quantitatively, due to the fact that at the stationary point of the effective potential the higher order terms become as important as lower order terms and hence the loop expansion breaks down. Therefore the result in this paper could at best be regarded as indicative. The final answer has to be settled by a non-perturbative means, such as the lattice simulation as mentioned in the Introduction.

In fact, a lattice generalization of the background field method is rather straightforward. Let us consider

$$
Z_{L}\left[J_{\mu}(x), B_{\mu}(x)\right] \equiv \int d U_{\mu}(x) \exp \left\{-S\left[U_{\mu}(x) B_{\mu}(x)\right]+\operatorname{Tr} J_{\mu}(x) f\left[U_{\mu}(x)\right]\right\}
$$

where $U_{\mu}(x)$ is the standard link variable, $B_{\mu}(x)$ is the background link variable, $S$ is the usual Wilson lattice action, $J_{\mu}(x)$ is a matrix valued external current and $f[U]$ is an arbitrary function satisfying $g f[U] g^{\dagger}=f\left[g U g^{\dagger}\right]$ for any unitary matrix $g$. Using the property of the invariance under an unitary transformation for the Haar measure one can easily verify the following

$$
\left.Z_{L}\left[\tilde{J}_{\mu}(x), \tilde{U}_{\mu}(x)\right]\right|_{\tilde{U}_{\mu}(x)=g(x) U_{\mu}(x) g^{\dagger}(x+\mu), \tilde{J}_{\mu}(x)=g(x) J_{\mu}(x) g^{\dagger}(x)}=Z_{L}\left[J_{\mu}(x), U_{\mu}(x)\right],
$$

a lattice version of Eq.2.2). A Legendre transform of $Z_{L}\left[J_{\mu}, U_{\mu}\right]$ would lead to a gauge invariant effective action, provided that the induced gauge field is constrained to have zero expectation value by adjusting $J_{\mu}(x)$, just as in the continuum case. The remarkable thing here is that we do not need to fix the gauge on a lattice and therefore the resulted effective action is unique for a given choice of the functional form of $f$.

As mentioned in the Introduction the background field ansatz Eq. (3.1) can be conveniently realized on a lattice. The constant nature of the ansatz avoids problems with the boundary condition and non-uniformness of the lattice constant effect due to the linear rising ansatz of Savvidy. Since Eq.2.2) only involves one parameter it may not be difficult to find a way to adjust the external current $J_{\mu}$ to ensure a vanishing of the expectation value for the induced quantum field. Work along this line will be pursued in the future.

\section{ACKNOWLEDGMENTS}

It is our pleasure to thank H. B. Nielsen, H. Trottier, P. van Baal, and especially Ken Johnson and Janos Polonyi for many very fruitful suggestions and discussions. This work is supported in part by funds provided by the U. S. Department of Energy (D.O.E.) under contracts \#DE-FG06-88ER40427, \#DE-AC02-76ER03069 and \#DE-FG0291ER40676.

\section{APPENDIX:}

In this appendix we explicitly calculate the divergent part of the effective potential for the background field Eq.(1.2) and show that the $\beta$-function remains the same as calculated with zero background field in four dimensions. Since we do not expect the divergent part to depend on the gauge choice in the Minimal subtraction scheme [19], we will pick the Feynman gauge $(\alpha=1)$ for convenience. 
The four-dimensional analog of $M(h ; p)$, Eq.(3.5), in the background field Eq.(1.2), is given by

$$
M(h ; p)=\operatorname{det}\left[M_{00}^{a b}(h ; p)\right] \cdot \operatorname{det}\left[M_{i j}^{a b}(h ; p)\right],
$$

with (again $m \equiv g h)$

$$
\begin{aligned}
\operatorname{det}\left[M_{00}^{a b}(h ; p)\right]= & {\left.\left[p^{2}+2 m^{2}\right]\left[\left(p^{2}+2 m^{2}\right)^{2}-4 m^{2} \mathbf{p}^{2}\right)\right], } \\
\operatorname{det}\left[M_{i j}^{a b}(h ; p)\right]= & {\left[p^{4}-4 m^{2} \mathbf{p}^{2}\right]\left[\left(p^{4}+4 m^{2} p^{2}\right)^{2}-4 m^{2} \mathbf{p}^{2}\left(p^{2}+2 m^{2}\right)^{2}\right] \times } \\
& \times\left[p^{6}+10 m^{2} p^{4}-4 m^{2} \mathbf{p}^{2} p^{2}+24 m^{4} p^{2}-8 m^{4} \mathbf{p}^{2}\right],
\end{aligned}
$$

while the ghost determinant $G(h ; p)=\operatorname{det}\left[M_{00}^{a b}(h ; p)\right]$. To regularize the momentum integration we use the finitetemperature extension of the usual dimensional regularization

$$
\int[d p] f(p) \equiv \frac{1}{\beta} \mu^{2 \epsilon} \int \frac{d^{3-2 \epsilon} \mathbf{p}}{(2 \pi)^{3-2 \epsilon}}\left[f\left(p_{0}=0, \mathbf{p}^{2}\right)+2 \sum_{n=1}^{\infty} f\left(p_{0}=\omega_{n} \equiv \frac{2 \pi n}{\beta}, \mathbf{p}\right)\right] .
$$

Since an overall additive constant of $V_{\text {eff }}(h)$ is irrelevant we can write

$$
V_{\mathrm{eff}}(h) \equiv \int_{0}^{m^{2}} d m^{2} \frac{\partial V_{\mathrm{eff}}(h)}{\partial m^{2}} .
$$

To carry out the spatial momentum integration it is convenient to rewrite

$$
\begin{aligned}
& \operatorname{det}\left[M_{00}^{a b}(h ; p)\right]=\prod_{l=1}^{3}\left[\mathbf{p}^{2}+a_{l}\left(m^{2}, \omega_{n}^{2}\right)\right], \\
& \operatorname{det}\left[M_{i j}^{a b}(h ; p)\right]=\prod_{l=4}^{12}\left[\mathbf{p}^{2}+a_{l}\left(m^{2}, \omega_{n}^{2}\right)\right],
\end{aligned}
$$

where the $a_{l}$ 's can be solved from Eq.(5.2) and (5.3). The explicit expressions of $a_{l}$ 's are too lengthy to be presented here. However, if our interest is to calculate the divergent part (or $1 / \epsilon$ term) we only need to notice that $a_{l}$ 's can be expanded as a power series of $m$ (when $n \neq 0$ ). In terms of $a_{l}$ 's the one-loop part of the effective potential becomes

$$
\frac{\partial V_{\mathrm{eff}}^{1-\mathrm{loop}}(h)}{\partial m^{2}}=\frac{1}{2} \sum_{l=1}^{12} s_{l} \int[d p] \frac{1}{\mathbf{p}^{2}+a_{l}\left(m^{2}, \omega_{n}^{2}\right)} \frac{\partial a_{l}\left(m^{2}, \omega_{n}^{2}\right)}{\partial m^{2}},
$$

with $s_{l}=-1$ for $l=1,2,3$ and $s_{l}=+1$ for $l=4,5, \ldots, 12$. The spatial momentum integration can be done trivially, yielding

$$
\frac{\partial V_{\mathrm{eff}}^{1-\mathrm{loop}}(h)}{\partial m^{2}}=\frac{\mu^{2 \epsilon} \Gamma(\epsilon-1 / 2)}{2 \beta(4 \pi)^{3 / 2-\epsilon}} \sum_{l=1}^{12} \frac{s_{l}}{3 / 2-\epsilon} \frac{\partial}{\partial m^{2}}\left[a_{l}\left(m^{2}, 0\right)^{3 / 2-\epsilon}+2 \sum_{n=1}^{\infty} a_{l}\left(m^{2}, \omega_{n}^{2}\right)^{3 / 2-\epsilon}\right] .
$$

To isolate the pole term in $\epsilon$ we need to expand $a_{l}\left(m^{2}, \omega_{n}^{2}\right)^{3 / 2-\epsilon}$ in a power series of $m / \omega_{n}$ in the infinite Matsubara frequency sum,

$$
a_{l}\left(m^{2}, \omega_{n}^{2}\right)^{3 / 2-\epsilon}=\omega_{n}^{3-2 \epsilon}\left[1+\sum_{i=1}^{\infty} a_{l}^{(i)}\left(\frac{m}{\omega_{n}}\right)^{i}\right],
$$

because $a_{l}\left(0, \omega_{n}\right)=\omega_{n}^{2}$. In general, the $a_{l}^{(i)}$,s are complex, but analytic in $\epsilon$. By noticing the fact that the Riemann zeta function $\zeta(s) \equiv \sum_{n=1}^{\infty} n^{-s}$ is singular only at $s=1$, we merely need to know $a_{l}^{(4)}$, which corresponds to $\omega_{n}^{-1-2 \epsilon}$ terms. An explicit calculation gives $a_{1}^{(4)}=3 / 2, a_{2}^{(4)}=a_{3}^{(4)}=-9 / 8, a_{4}^{(4)}=a_{5}^{(4)}=15 / 8, a_{6}^{(4)}=a_{7}^{(4)}=3 / 2, a_{8}^{(4)}=a_{9}^{(4)}=15 / 8$, $a_{10}^{(4)}=-9 / 2$ and $a_{11}^{(4)}=a_{12}^{(4)}=39 / 8$. So the expression for the divergent part of the effective potential becomes

$$
\begin{aligned}
V_{\mathrm{eff}}^{1-\text { loop }, \mathrm{div}}(h) & =\frac{\mu^{2 \epsilon} \Gamma(\epsilon-1 / 2)}{\beta(4 \pi)^{3 / 2-\epsilon}} \sum_{l=1}^{12} \frac{s_{l} a_{l}^{(4)}}{3 / 2-\epsilon} m^{4}\left(\frac{\beta}{2 \pi}\right)^{1+2 \epsilon} \zeta(1+2 \epsilon) \\
& =-\frac{g^{4} h^{4}}{24 \pi^{2} \epsilon} \sum_{l=1}^{12} s_{l} a_{l}^{(4)}
\end{aligned}
$$


which is independent of $\beta$ (temperature) and real, as it should be. Using the explicit values of $a_{l}^{(4)}$ 's and adding the classical contribution $3 g^{2} h^{4} / 2$, we finally have

$$
V_{\text {eff }}(h)=\frac{3}{2} g^{2} h^{4}\left[1-\frac{11 g^{2}}{24 \pi^{2} \epsilon}\right]+\text { finite terms } .
$$

Due to the explicit gauge invariance of the effective potential in the background field approach, the product $g h$ needs no renormalization [9]. Hence the factor in the bracket in the above equation is nothing but $Z_{q}^{2}$, which leads to the correct $\beta$-function. Therefore, the ultraviolet behavior of the $\mathrm{SU}(2)$ Yang-Mills theory in the background Eq.(1.2) remains universal.

[1] G. K. Savvidy, Phys. Lett. 71B, (1977) 133.

G. Matinyan and G. K. Savvidy, Nucl. Phys. B134 (1978) 539.

H. Pagels and E. Tomboulis, Nucl. Phys. B143 (1978) 485.

[2] N. K. Nielsen and P. Olesen, Nucl. Phys. B144 (1978) 376.

[3] H.B. Nielsen and M. Ninomiya, Nucl. Phys. B156 (1979) 1;

J. Ambjørn and P. Olesen, Nucl. Phys. B170 (1980) 60 and 265;

J. Ambjørn , B. Felsager and P. Olesen, Nucl. Phys. B175 (1980) 349.

[4] L. Maiani, G. Martinelli, G. C. Rossi and M. Testa, Nucl. Phys. B273 (1986) 275.

[5] J. Ambjørn et al., Phys. Lett. 225B (1989) 153; Phys. Lett. 245B (1990) 575.

P. Cea and L. Cosmai, Phys. Rev. D43 (1991) 620; Phys. Lett. 264B (1991) 415.

A. R. Levi and J. Polonyi, MIT preprint, CTP\#2161, March 1993.

[6] H. D. Trottier and R. M. Woloshyn, Phys. Rev. Lett. 70 (1993) 2053;

P. Cea and L. Cosmai, preprint BARI-TH 129/93.

[7] K. Johnson, in QCD - 20 Years Later, Aachen. June 1992;

D. Z. Freedman, P. E. Haagensen, K. Johnson and J. I. Latorre, MIT preprint, CTP\#2238.

[8] L. S. Brown and W. I. Weisberger, Nucl. Phys. B157 (1979) 285.

[9] B. S. DeWitt, Phys. Rev. 162 (1967) 1195;

J. Honerkamp, Nucl. Phys. B36 (1971) 130;

G. 't Hooft, Nucl. Phys. B62 (1973) 444;

L. F. Abbott, Nucl. Phys. B185 (1981) 189;

D. G. Boulware, Phys. Rev. D23 (1981) 389.

[10] G. A. Vilkovisky, Nucl. Phys. B234 (1984) 125; A. O. Barvinsky and G. A. Vilkovisky, Phys. Rep. 119 (1985) 1.

[11] T. H. Hansson and I. Zahed, Nucl. Phys. B292 (1987) 725;

M. E. Carrington, T. H. Hansson, H. Yamigishi and I. Zahed, Ann. Phys. 190 (1989) 373.

[12] A. Salam and J. Strathdee, Nucl. Phys. B90 (1975) 203.

[13] J. Schwinger, Phys. Rev. 82 (1951) 664.

[14] H. D. Trottier, Phys. Rev. D44 (1991) 464.

[15] R. Parthasarathy, M. Singer and K. S. Viswanathan, Can. J. Phys. 61 (1983) 1442.

[16] M. Lüscher, Nucl. Phys. B219 (1983) 233;

M. Lüscher and G. Münster, Nucl. Phys. B232 (1984) 445.

[17] J. Koller and P. van Baal, Ann. Phys. 174, (1987) 299; Nucl. Phys. B302 (1988) 1.

[18] M. Lüscher, R. Narayanan, P. Weisz and U. Wolff, Nucl. Phys. B384 (1992) 168.

[19] D. J. Gross, in "Methods in Field Theory", eds. R. Balian and J. Zinn-Justin, North-Holland, (1976). 